Journal of Applied Fluid Mechanics, Vol. 9, Special Issue 1, pp. 49-57, 2016.

Selected papers from the $7^{\text {th }}$ International Exergy, Energy

and Environment Symposium, IEEE7-2015

Available online at www.jafmonline.net, ISSN 1735-3572, EISSN 1735-3645.

DOI: 10.36884 /jafm.9.SI1.25826

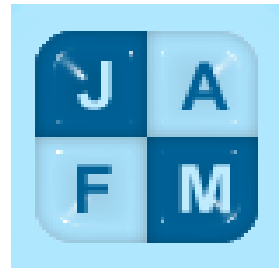

\title{
Numerical and Experimental Study on the Effects of Taylor Number on the Wavelength of the Couette-Taylor Flow

\author{
M. Monfared ${ }^{1 \dagger}$, E. Shirani ${ }^{2}$, F. Aloui ${ }^{3}$, M. R. Salimpour ${ }^{4}$ \\ ${ }^{1}$ Phd student, Department of Mechanical Engineering, Isfahan University of Technology, \\ Isfahan, 8415683111, Iran \\ ${ }^{2}$ Professor, Department of Mechanical Engineering, Foolad Institute of Technology, \\ Fooladshahr, Isfahan, 84916 63763, Iran \\ ${ }^{3}$ Professor, University of Lille North of France, University of Valenciennes (UVHC), \\ LAMIH CNRS UMR 8201, Department of Mechanics, Campus Mont Houy, \\ 59313 Valenciennes Cedex 9, France \\ ${ }^{4}$ Associate Professor, Department of Mechanical Engineering, Isfahan University of Technology, \\ Isfahan, 8415683111, Iran
}

†Corresponding Author Email: m.monfared@me.iut.ac.ir

(Received October 20, 2015; accepted December 10, 2015)

\begin{abstract}
In the present study, the hydrodynamic behaviours of the Couette Taylor flow in different flow regimes were investigated, experimentally and numerically.In this research, the effects of Taylor number on wavelength of the flow, which are two important hydrodynamic charactristics of the Couette Taylor flow, are investigated both experimentally and numerically in order to study the stability of the flow and formation of vortices. In addition, the velocity and pressure variation of the the flow between two cylinders were considered and compared for different Taylor numbers to understand the behaviuor of the flow.
\end{abstract}

Keywords: Couette-Taylor flow, wavelength, Taylor number, stability.

\section{NOMENCLATURE}

$\begin{array}{ll}\mathrm{R} & \text { Cylinder radius } \\ \mathrm{P} & \text { Pressure } \\ \mathrm{d} & \text { Heigth of cylinder } \\ \mathrm{t} & \text { Time } \\ \mathrm{k} & \text { Turbulent kinetic energy } \\ \mathrm{Gk} & \text { Generation of turbulent kinetic energy } \\ \mathrm{G} \omega & \text { Generation of dissipation rate } \\ \mathrm{u} & \text { Velocity } \\ \mathrm{v} & \text { Velocity } \\ \mathrm{Ta} & \text { Taylor number } \\ \mathrm{Re} & \text { Reynolds number } \\ \mathrm{D} & \text { Effective diffusivity } \\ \mathrm{x} & \text { Coordinate } \\ \mathrm{y} & \text { Coordinate }\end{array}$

\section{INTRODUCTION}

The present study is related to the numerical modelling and experimental investigation of fliud flow which is confined between two coaxial cylinders, with the inner cylinder is rotating and outer one is stationary. The geometrical simplicity of the Couette-Taylor systems has encouraged the researchers and the industrials to investigate in this
Greek letters.

$\begin{array}{ll}\Omega & \text { Rotational speed } \\ \eta & \text { Radial ratio } \\ \Gamma & \text { Aspect ratio } \\ \delta & \text { Curvature ratio } \\ \rho & \text { Density } \\ \mathrm{Y} & \text { Dissipation } \\ \omega & \text { Dissipation rate } \\ \lambda & \text { Wavelength } \\ \text { Subscripts } \\ 1 & \text { Inner cylinder } \\ 2 & \text { Outer cylinder } \\ \mathrm{k} & \text { Turbulent kinetic Energy } \\ \omega & \text { Dissipation } \\ \mathrm{c} & \text { Critical value }\end{array}$

kind of systems. Indeed, Couette-Taylor systems is widely used as reactor and bioreactor, filtration process and photocatalytic reactor for water treatment, to establish the optimum conditions for lubrication of the transmission speed stages in the car industries, in aeronautics and the power stations of electrical energy production, etc. (Cohen et al., 1991; Holeschovsky et al., 1991). 
The circular motion of a fluid in concentric rotating cylinders is described by Newton in 1687, (Newton, 1934). Stokes was the first to consider this flow theoretically in 1848 noting the difficulty in the boundary conditions at the walls of the cylinder, now known as the no-slip boundary condition. He indicated that, if the inner cylinder were made to revolve too fast, eddies would be produced (Stokes, 1880). This remarkable point of view was made many years before vortices were visualized. When only the outer cylinder rotates or the rotation rate of the inner cylinder is blow a critical value, the flow is stable and no vortical flow is observed.

Mallock and Couette independently used two differentially rotating concentric cylinders to measure the fluid viscosity. Couette rotated the outer cylinder only while the inner cylinder is fixed. In this case, flow is stable and no vertical structures appear (Couette, 1890). Mallock also rotated the inner cylinder and kept the outer cylinder fixed. The results were the production of Taylor vortices (Mallock, 1888). Based on these observations, Rayleigh analysed the inviscid flow between to concentric cylinders when the inner cylinder is rotated and explained the physical origin of the vortical structure. He used inviscid approach to the instability based on an imbalance of the centrifugal force and pressure gradient force. He argued that if the radial component of momentum decreases in the radial direction, as it does for an inner rotating cylinder and a fixed outer cylinder, the flow is unstable (Rayleigh, 1916).

Finally Taylor was able to relate theory and experiment for stability in cylindrical Couette flow using linear stability theory to Navier Stokes equations for viscous flow with no slip boundary conditions. He accurately predicted experimental results of transition from stable flow to vortical Taylor-Couette flow. Taylor showed that based on small perturbations of the velocity and pressure fields, how viscosity stabilizes the flow at low rotational speeds using linear stability analysis. He introduced the critical Taylor number, Tcrit, a dimensionless number defined as the ratio of centrifugal (or inertial) to viscous forces. Below the critical Taylor number, the flow is stable with no vortical structure, above it the flow is unstable with toroidal vortices (Taylor, 1923).

Couette-type flows are known as the flows which are driven by the motion of one wall bounding a viscous liquid. Vortical Taylor-Couette flow is formed due to the shear flow between a rotating inner cylinder and a concentric, fixed outer cylinder when the flow becomes unstable. The instability occurs as the rotation of the inner cylinder increases resulting in pairs of counter-rotating, axisymmetric, toroidal vortices (Taylor vortices). These vortices fill the annulus and are superimposed on the Couette flow. Each pair of vortices has a wavelength of approximately twice of the clearance between to cylinders. The axial and radial components of velocity related to the Taylor vortices are relatively small and only a few percent of the linear speed of the inner cylinder wall (Wereley et al., 1998).
Up to now, only very few works have been dedicated to real numerical modelling of complicated turbulent flow in such swirl flows especially to simulate the flow from laminar to turbulent flow and to study and model the transition process from laminar to turbulent flows (Dong, 2008; Bazilevs et al., 2010; Berrich, 2011; Berrich et al., 2012; Poncet, 2013). In this research, an experimental set up and numericall model were designed to study the Couette-Taylor flow. So the Taylor number and wavelength of the flow are investigated from laminar to turbulent flow regime in order to study the stability and the behaviuor of the flow in different regimes. Also the effects of Taylor number on velocity and pressure variation of the flow between two cylinders are investigated too.

\section{EXPERIMENTAL FACILITY}

\subsection{Setup}

The experimental installation is shown is Figure 1. It is composed of an inner cylinder with a radius $\mathrm{R} 1$ $=85.5 \pm 0.2 \mathrm{~mm}$ and an outer cylinder with a radius $\mathrm{R} 2=100 \pm 0.2 \mathrm{~mm}$. The corresponding radius ratio is $\eta=0.855$ and the aspect ratio is $\Gamma=31$. The whole apparatus is made in Plexiglas to assure the Couette-Taylor flow visualization study. The inner cylinder was driven by an $\alpha$-Step electrical motor. The engine imposed to the mobile inner cylinder a maximum speed of $120 \mathrm{rpm}$. It was supplied by a signal generator. The revolutions were controlled by a speed controller.

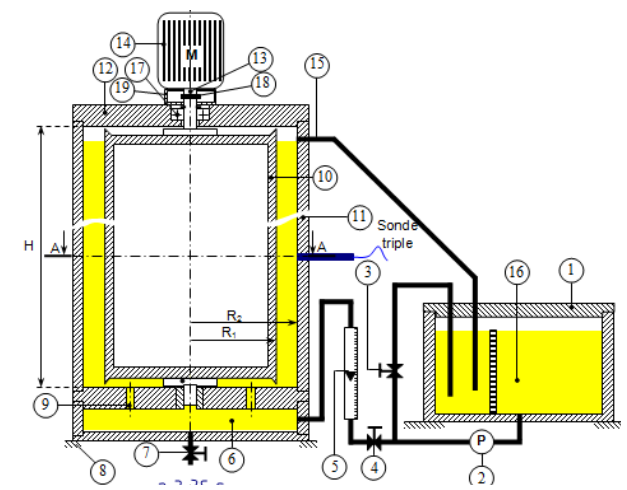

Fig. 1. Experimental installation used by Berrich (2011)

1 Storage tank; 2 Pump; 3 By-pass; 4 Flowrate control valve; 5 Flowmeter; 6 CT feeder tank; 7 Drain valve; 8 Support; 9 Supply holes for the homogeneous filling of the CT gap (8 holes); 10

CT inner cylinder; 11 CT outer cylinder; 12 Flabby; 13 Motor shaft; 14 Electrical motor; 15 Overflow; 16 Ball bearing; 17 Elastic coupling; 18 Protection; 19 Circular probe.

The Kalliroscope is generally used to visualize flows. It is a suspension of reflective particles which having a density of $1.62 \mathrm{~g} / \mathrm{cm} 3$. It has a bidimensional anisotropy. The Kalliroscope particles dispersed in a solution (approximately $1 \%$ to $2 \%$ ) have a strong refraction index equals to 1.85 . Thus, they are easily visible, even in strongly diluted 
solutions (lower than $0.1 \%$ ). The particles are aligned in the current lines direction revealing thus the flow structure. The flow is visible if the particle has time to be suitably directed. The visualizations using Kalliroscope particle were conducted using a CCD camera with a resolution of $1600 \times 1200$ pixels.

\subsection{Measurements}

Direct visualization of the Couette flow by particles Kalliroscope is shown in Figure 2. It is represented a spatial homogeneity the intensity of the reflected light. Also the basic flow is perfectly homogeneous along the $\mathrm{z}$ axis.
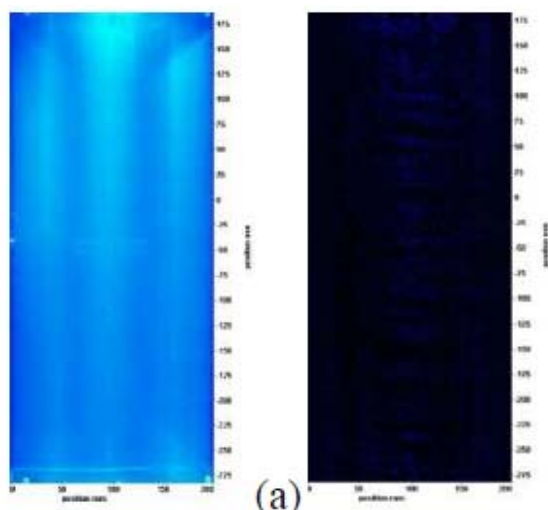

(a)

Fig. 2. Visualization of Couette flow according to (Berrich, 2011): a) Base flow visualization; b) Uniform distribution of particles Kalliroscope for Couette flow

By increasing the Taylor number, the evolution of the intensity of the reflected light becomes spatially inhomogeneous. Field intensity of the light reflected by the Kalliroscope particles are illustrated in Figure 3. So the vortices are appeared at $\mathrm{Ta}=45.45$. This value of the Taylor number named critical Taylor number. Hence the first transition created and the flow regime named Taylor vortex flow. In addition, the flow is periodic with wavelength equal to $\lambda=29 \mathrm{~mm}$.
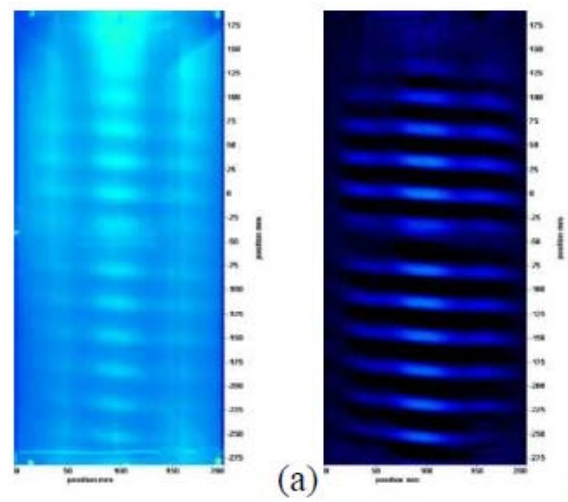

Fig. 3. Taylor vortex regime for $\mathrm{Ta}=\mathbf{4 5 . 4 5}$ (Berrich, 2011): a) Flow visualization rolls Taylor in Taylor vortex regime

b) Kalliroscope particle distribution
The second instability corresponds to the appearance of the azimuthal wave which is superimposed on the Taylor vortices generating their swing. Visualizing the flow of particles is Kalliroscope shown in Figure 4. The appearance of the second instability at $\mathrm{TaC} 2=60.60$, characterized by wavy vortex form networks that are corrugated in the axial direction and in the direction azimuth. Wavy vortex is periodic in the axial direction with a period $\lambda$, which is equal to $36.25 \mathrm{~mm}$ for this flow regime.

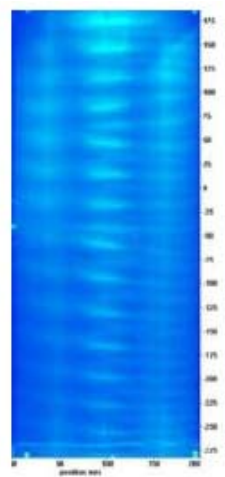

(a)

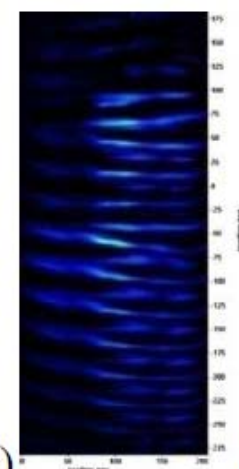

(b)

Fig. 4. Wavy vortex flow regime for $\mathrm{Ta} \approx 60$ (Berrich, 2011): a) flow visualization Taylor curled in a wavy vortex flow

b) Kalliroscope particle distribution

\section{NUMERICAL SIMULATION}

\subsection{Geometry and Boundary Conditions}

The geometry is a concentric cylinder, which the inner cylinder is rotating while the outer cylinder is stationary, and the fluid between the cylinders is water. The diameters of these external and internal cylinders are respectively $200 \mathrm{~mm}$ and $171 \mathrm{~mm}$ with the height of $450 \mathrm{~mm}$ (see Figure 5). Also, no-slip boundary condition is imposed on the solid boundaries.

Also other parameters for Couette-Taylor systems are:

$$
\begin{aligned}
& d=R_{2}-R_{1}=14.5 \mathrm{~mm} \\
& \eta=\frac{R_{1}}{R_{2}}=0.855 \\
& \Gamma=\frac{h}{d}=31 \\
& \delta=\frac{d}{R_{1}}=0.1696
\end{aligned}
$$

\subsection{Governing Equations and Turbulence Modeling}

The Couette-Taylor flow is governed by continuity and momentum equations. In this survey, the standard k- $\omega$ model is applied in order to simulate the flow. This model showed the good agreement with experimental data in the past considerations. 


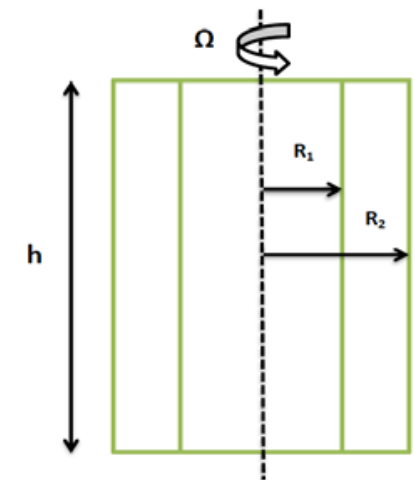

a) Front view

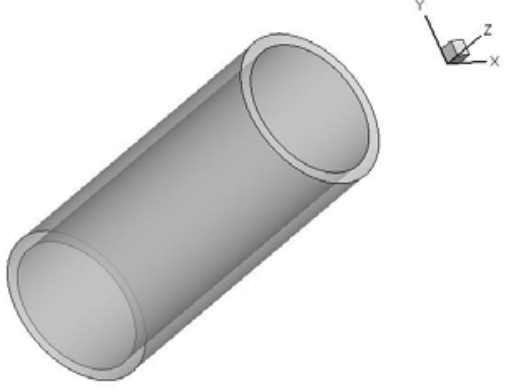

b) 3-D view

Fig. 5. Schematic illustration of Couette Taylor system

The standard k- $\omega$ model is based on the Wilcox $k-\omega$ model, which incorporates modifications for lowReynolds number effects, compressibility, and shear flow spreading.

The standard k- $\omega$ model is an empirical model based on model transport equations for the turbulence kinetic energy $(\mathrm{k})$ and the specific dissipation rate $(\omega)$.

The turbulence kinetic energy, $k$, and the specific dissipation rate, $\omega$, are obtained from the following transport equations:

$$
\frac{\partial}{\partial t}(\rho k)+\frac{\partial}{\partial x_{i}}\left(\rho k u_{i}\right)=\frac{\partial}{\partial x_{j}}\left(D_{k} \frac{\partial k}{\partial x_{j}}\right)+G_{k}-Y_{k}
$$

and

$$
\frac{\partial}{\partial t}(\rho \omega)+\frac{\partial}{\partial x_{i}}\left(\rho \omega u_{i}\right)=\frac{\partial}{\partial x_{j}}\left(D_{\omega} \frac{\partial \omega}{\partial x_{j}}\right)+G_{\omega}-Y_{\omega}
$$

\subsection{Grid Generation}

After designing the Couette-Taylor model, the grid should be generated to solve the Couette-Taylor flow. Hence, the structured grid, because of high accuracy and fast convergency, is generated for solving the flow equations. Figure 6 showed the computational grid system that is employed in this study.

\subsection{Grid study}

At first, the grid study was done to obtain the suitable number of grid cells. The variation of the

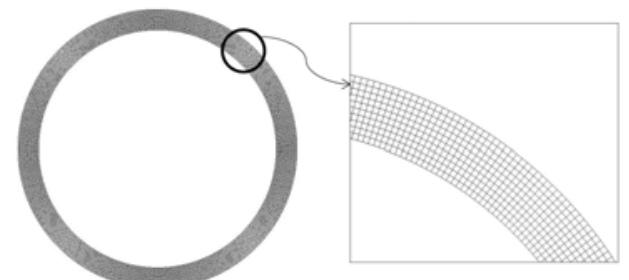

a) Front view

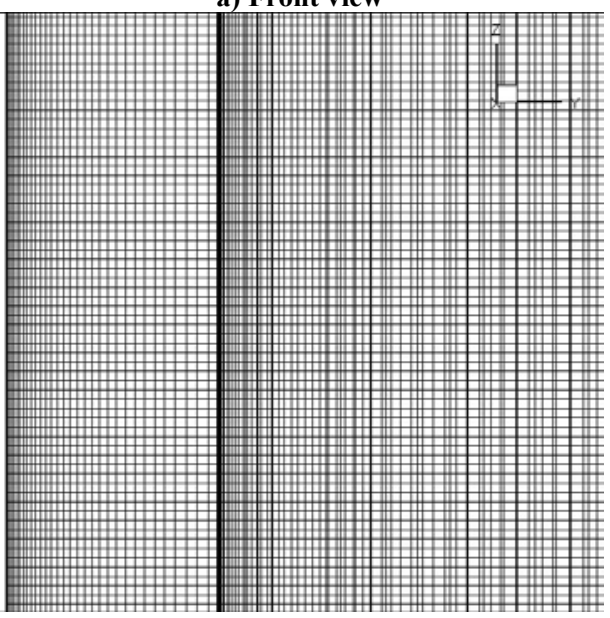

b) Longitudinal direction

Fig. 6. Numerical grid for Couette Taylor system

velocity and pressere of the flow between two cylinders are compared to choose the best number of grid cells. The Taylor and Reynolds numbers, for different number of grid cells are given in table 1 .

Figures 7 and 8 illustrated the velocity and pressure profiles respectively. The results showed that by increasing the number of grid cells, the differences between profiles decrease. The differences for velocity and pressure profiles for $\mathrm{N}=2,800,000$ and $\mathrm{N}=3,350,000$ are less than $1 \%$, so the number of grid cells $\mathrm{N}=2,800,000$ is a suitable selection for simulation.

Table 1 Flow parameters for grid study

\begin{tabular}{|c|c|c|c|}
\hline Ta & Re & $\begin{array}{c}\text { Rotation } \\
\text { speed (rad/s) }\end{array}$ & $\begin{array}{c}\text { Number of } \\
\text { grid cells }(\mathrm{N})\end{array}$ \\
\hline \multirow{3}{*}{1297.25} & \multirow{3}{*}{3150} & \multirow{3}{*}{2.55} & $\mathrm{~N}=1,400,000$ \\
\cline { 4 - 4 } & & & $\mathrm{N}=2,200,000$ \\
\cline { 4 - 4 } & & $\mathrm{N}=2,800,000$ \\
\cline { 3 - 4 } & & $\mathrm{N}=3,350,000$ \\
\hline
\end{tabular}

\subsection{Validation of the numerical results}

After grid study, the present numerical results and experimental data are compared in order to validate the present numerical simulation. The critical Taylor number for transition of the flow from Couette regime to Taylor vortex regime and then from Taylor vortex regime to wavy vortex regime are compared. Also the wavelengths of these two regions of the flow are also compared.

These comparisons are represented in tables 2 and 3. It is concluded that, the present numerical results have good agreements with the experimental data. 


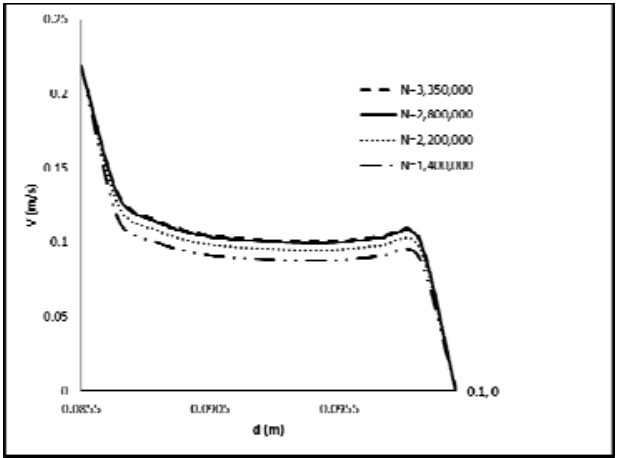

Fig. 7: Velocity profile for flow between coaxia cylinders

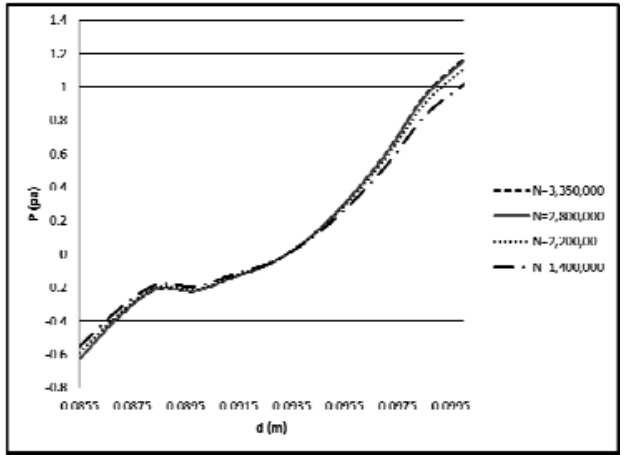

Fig. 8: Pressure profile for flow between coaxial cylinders

Tab 2 Critical Taylor numbers of experimental data and numerical simulation

\begin{tabular}{|l|l|l|l|}
\hline \multirow{2}{*}{ Flow pattern } & Ta & Dafference \\
\cline { 2 - 3 } & $\begin{array}{l}\text { Numerica } \\
1\end{array}$ & $\begin{array}{l}\text { Experimenta } \\
1\end{array}$ & $\begin{array}{l}\text { Desults } \\
\text { Data } \\
\%\end{array}$ \\
\hline $\begin{array}{l}\text { Transition from } \\
\text { Couette flow to Taylor vortex flow }\end{array}$ & 45.3 & 45.45 & 0.33 \\
\hline $\begin{array}{c}\text { Transition from } \\
\text { Taylor vortex flow to wavy vortex flow }\end{array}$ & 59.72 & 61.45 & 1.45 \\
\hline
\end{tabular}

Table 3 Wavelength obtained from the experimental data and numerical simulation

\begin{tabular}{|c|l|l|l|}
\hline Flow pattern & \multicolumn{2}{|l|}{$\lambda=$ Wavelength $(\mathrm{mm})$} & \multirow{2}{*}{$\begin{array}{c}\text { Differences } \\
\text { numerical }\end{array}$} \\
\cline { 2 - 3 } & $\begin{array}{l}\text { Experimental } \\
\text { data }\end{array}$ & \\
\hline $\begin{array}{c}\text { results } \\
\text { flow vor vortex }\end{array}$ & 29.23 & 29 & 0.79 \\
\hline $\begin{array}{c}\text { Wavy vortex } \\
\text { flow }\end{array}$ & 38.63 & 36.25 & 6.6 \\
\hline
\end{tabular}

\section{RESULTS AND DISCUSSIONS}

After validation of the numerical results, the flow is simulated for different flow regimes which include Couette flow, Taylor vortex flow, wavy vortex flow, modulated wavy flow and turbulent flow. Thus by increasing the speed of inner cylinders, the flow regimes change. The simulation was done from low Taylor numbers up to high Taylor numbers.

\subsection{Flow simulation}

At first, the flow is simulated for low Reynolds numbers or Taylor numbers. Base on the numerical results, when the Reynolds numbers are less than 110 (or Taylor numbers are less than 43) the flow regime is Couette flow and do not consist of any vortices in the flow.

The velocity and pressure distribution showed that, the flow is homogeneous with respect to $\mathrm{z}$ direction (see Figures 9 and 10). Also the velocity distribution is linear and the maximum and minimum values are on the inner and outer cylinder walls respectively. The results showed that, the vortices are not formed and damped by viscosity, except near of the top wall that due to wall effects, the small vortices are formed.

For better visualization, and due to symmetry, only

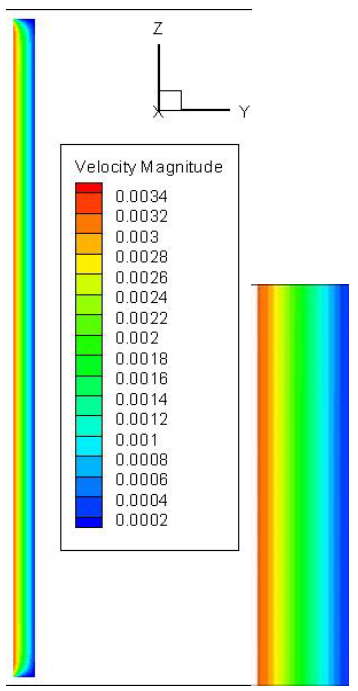

Fig. 9. The velocity distribution for Couette flow $(\operatorname{Re}=50, T a=20.59, \Omega=0.04052 \mathrm{rad} / \mathrm{s})$

half of the pictures of the numerical results are presented in all Figures.

Vortex rings are appeared in a threshold value of Taylor number (or Reynolds number) that known as a critical Taylor number Tac1 (or critical Reynolds number Rec1). The numerical results represented 


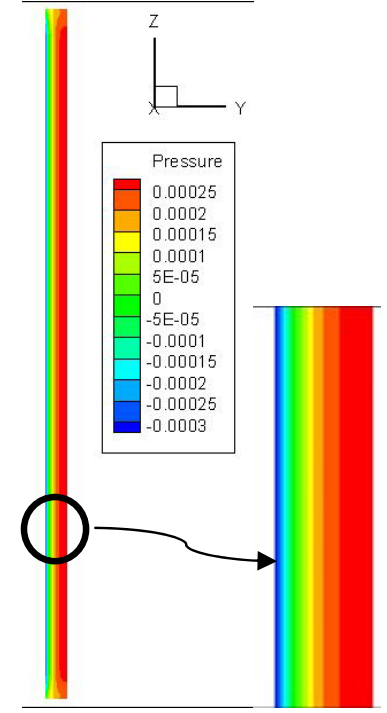

Fig. 10. The pressure distribution for Couett flow $(\operatorname{Re}=50, T a=20.59, \Omega=0.04052 \mathrm{rad} / \mathrm{s})$

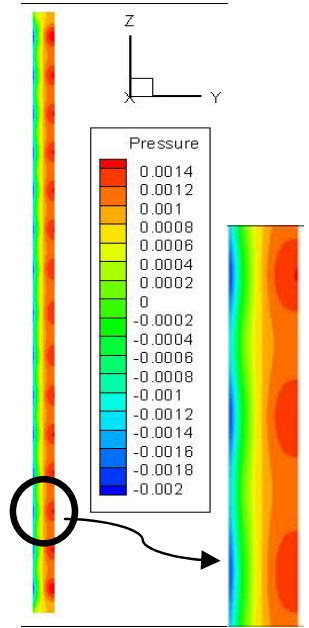

Fig. 12. The pressure distribution for Taylor vortex flow $(\operatorname{Re}=110, \mathrm{Ta}=\mathbf{4 5 . 3}, \Omega=0.08915 \mathrm{rad} / \mathrm{s})$

that approximately at the $\mathrm{Re}=110$ or $\mathrm{Ta}=45.3$, the vortices are formed. So the regime of flow changed from Couette flow into Taylor vortex flow.

The distribution of velocity and pressure and also streamlines of the flow domain represented that the vortices and fluctuations are formed (see Figures 11,12 and 13). So the pairs of counter-rotating, axisymmetric, toroidal vortices - that called "Taylor vortices" appeared in the flow and are superimposed on the Couette flow. The numerical results ilustrated that, the wavelength of the flow is equal to $\lambda=29.23 \mathrm{~mm}$ (see Figure 13).

By increasing the rotational speed of the inner cylinder, a second transition that known as a wavy vortex flow appears, so the formation and wave length of the vortices changed. In this section, the numerical results for $\mathrm{Re}=500 \quad(\mathrm{Ta}=206)$ are represented (see Figures 14 and 15). The numerical results showed that, the wavelength of the flow is equal to $\lambda=38.63 \mathrm{~mm}$ (see Figure 16).

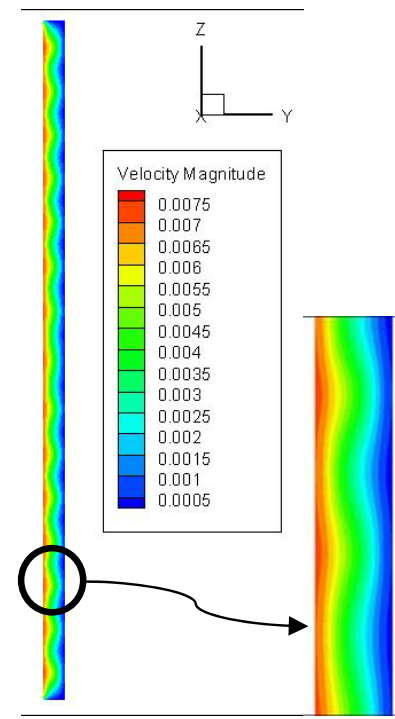

Fig. 11. The velocity distribution for Taylor vortex flow $(\operatorname{Re}=110, T a=45.3, \Omega=0.08915 \mathrm{rad} / \mathrm{s})$

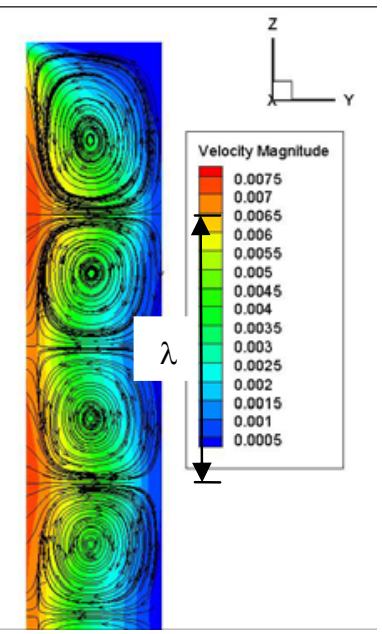

Fig. 13. Vortex formation and wavelength of the Taylor vortex flow $(\operatorname{Re}=110, \mathrm{Ta}=45.3, \Omega=0.08915$

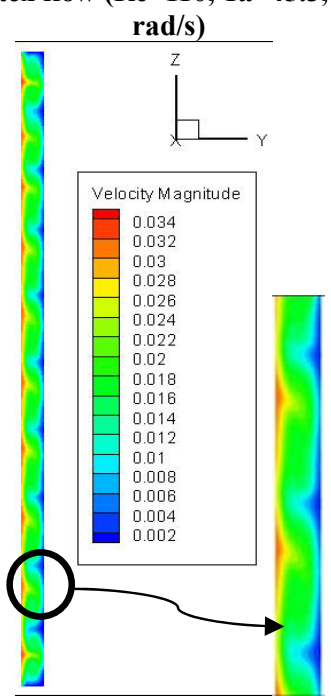

Fig. 14. The velocity distribution for Wavy vortex flow $(\operatorname{Re}=500, T a=206, \Omega=0.4052 \mathrm{rad} / \mathrm{s})$ 


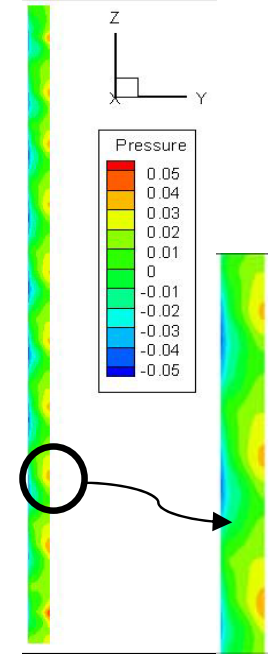

Fig. 15. The pressure distribution for Wavy vortex flow $(\operatorname{Re}=500, T a=206, \Omega=0.4052 \mathrm{rad} / \mathrm{s})$

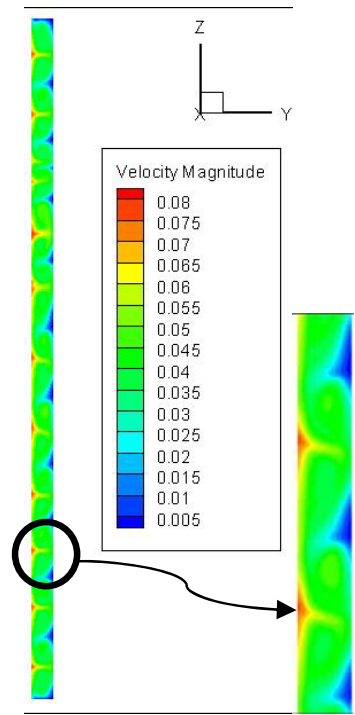

Fig. 17. The velocity distribution for modulated wavy vortex flow $(\operatorname{Re}=1250, T a=514.8, \Omega=1.013$ $\mathbf{r a d} / \mathbf{s}$ )

By increasing Taylor number, next transition that known as a modulated wavy vortex flow appears, so the formation and wave length of the vortices changed. In this section, the numerical results for $\mathrm{Re}=1250(\mathrm{Ta}=514.8)$ are illustrated (see Figures 17 and 18). The numerical results showed that, the wavelength of the flow is equal to $\lambda=39.6 \mathrm{~mm}$ (see Figure 19)

The turbulent regime appears at higher Taylor numbers. It is characterized by homogenization of the flow and better diffusion in the presence of turbulent structures. The results are investigated for $\mathrm{Re}=3150 \quad(\mathrm{Ta}=1297.25)$. The numerical results showed that, in this regime the wavelength of the flow is equal to $\lambda=42 \mathrm{~mm}$ (see Figure 22).

\subsection{Comparision of different flow regimes}

The numerical results for different Taylor or Reynolds numbers are compared in order to obtain

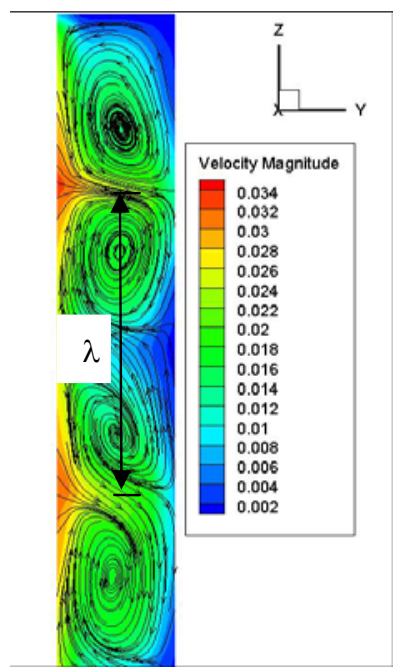

Fig. 16. Vortex formation and wavelength of the Wavy vortex flow $(\operatorname{Re}=\mathbf{5 0 0}, \mathrm{Ta}=\mathbf{2 0 6}, \Omega=\mathbf{0 . 4 0 5 2}$

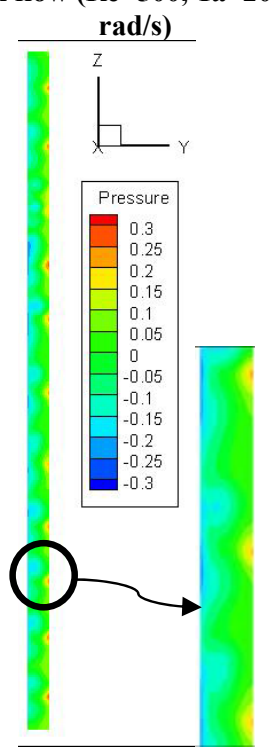

Fig. 18. The pressure distribution for modulated wavy vortex flow $(\operatorname{Re}=1250, T a=514.8, \Omega=1.013$ $\mathrm{rad} / \mathrm{s}$ )

better understanding with respect to Couette Taylor flow. Figures 23 and 24 represent the comparision among velocity variation for different flow regimes. It is observed that for low Taylor numbers, the velocity profile is linear, so it means that there are no vorticese in the flow. At $\mathrm{Ta}=45.3$, the velocity profile has a little deviation from linear form, hence it concluded that it is the onset of the vortices. So by increasing the Taylor numbers, the velocity profile deviated from linear form which is because of the formation of vortices in the flow. Also deviation from linear form increased by enhancing Reynolds numbers.In addition, Figures 25 and 26 shows the pressure distribution for different Taylor numbers. It is represented that at low Taylor numbers the pressures is mainly positive except near the inner cylinder wall, which is because of the higher velocity values. By formation of vortices and increasing the Taylor numbers, approximately half of the pressure values became negative. 


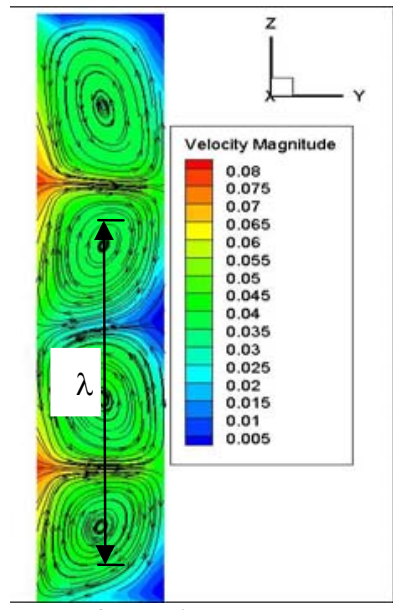

Fig. 19. Vortex formation and wavelength of the modulated wavy vortex flow $(\operatorname{Re}=1250, \mathrm{Ta}=514.8$, $\Omega=1.013 \mathrm{rad} / \mathrm{s}$ )

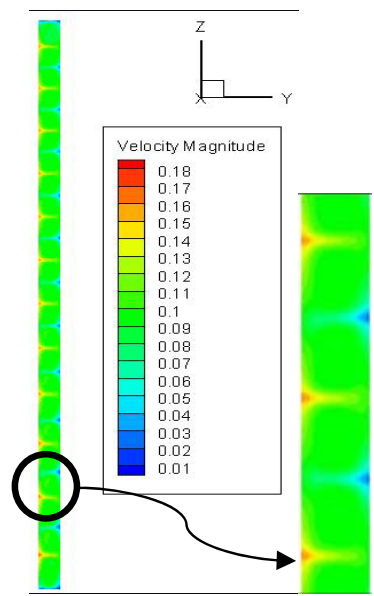

Fig. 20. The velocity distribution for turbulent vortex flow $(\operatorname{Re}=3150, \mathrm{Ta}=1297.25, \Omega=2.55 \mathrm{rad} / \mathrm{s})$

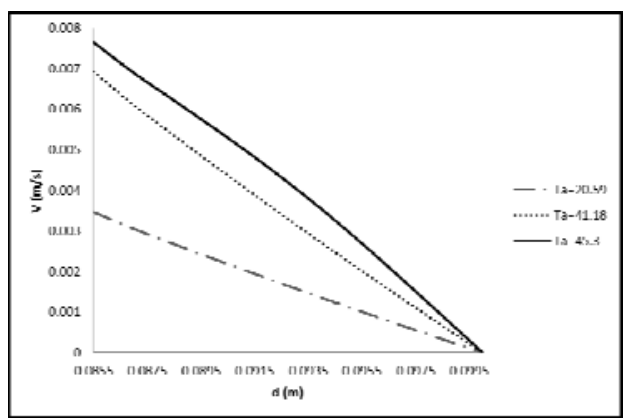

Fig. 23. Velocity profiles for different Taylor numbers

Also, Figure 27 represents the wavelength of the flow with respect to the Taylor number. It is observed that the wavelength of the flow increased dramatically at onset of Taylor vortex flow and then increased gradually at higher Taylor numbers.

\section{Conclusions}

The main goal of this research is to study of the wavelength of the Couette-Taylor flow at different

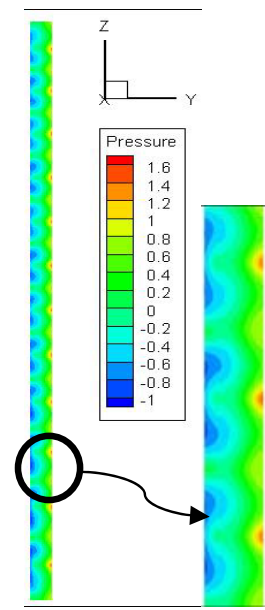

Fig. 21. The pressure distribution for turbulent vortex flow $(\operatorname{Re}=3150, T a=1297.25, \Omega=2.55 \mathrm{rad} / \mathrm{s})$

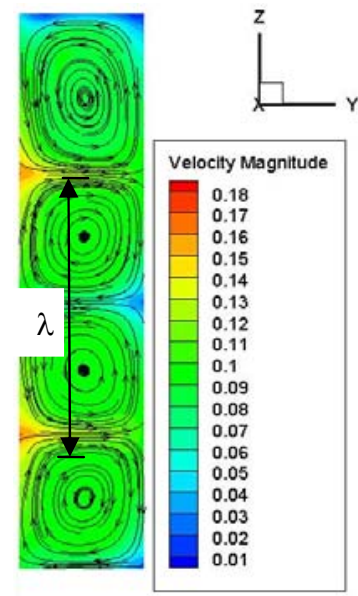

Fig. 22. Vortex formation and wavelength of the turbulent vortex flow $(\mathrm{Re}=3150, \mathrm{Ta}=\mathbf{1 2 9 7 . 2 5}$, $\Omega=2.55 \mathrm{rad} / \mathrm{s})$

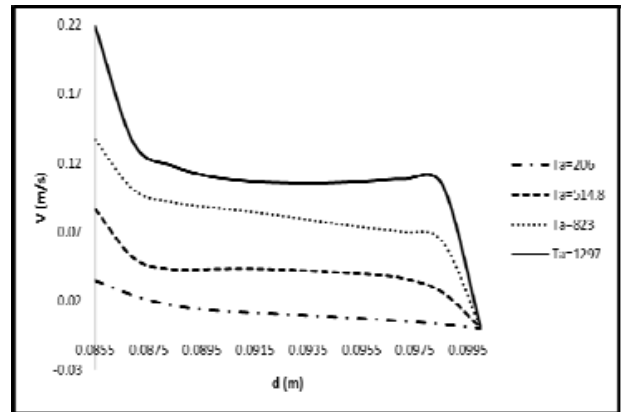

Fig. 24. Velocity profiles for different Taylor numbers

flow regimes, in order to investigate the behaviour and the structure of the flow. So the distribution of the velocities, pressures and also the formation of vortices are considered. The conclusions of this research are as follow:

The present numerical results have very good concurrence with the experimental data. So it concludes that the present modeling is valid for rotating flows. 


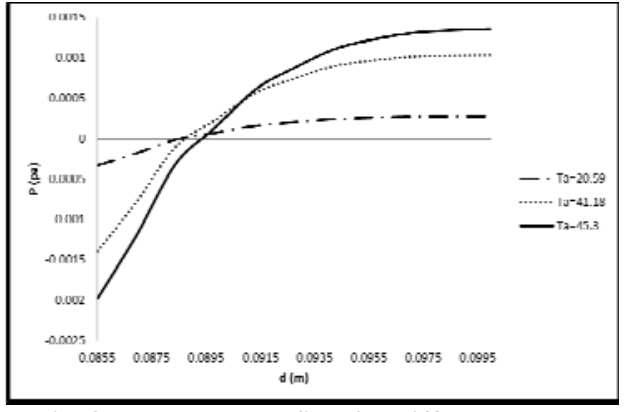

Fig. 25. Pressure profiles for different Taylor numbers

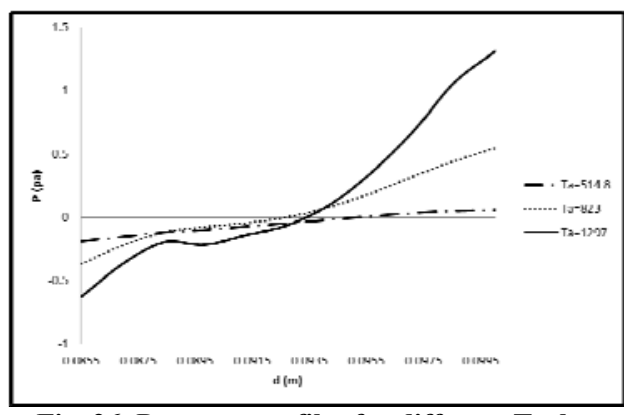

Fig. 26. Pressure profiles for different Taylor numbers

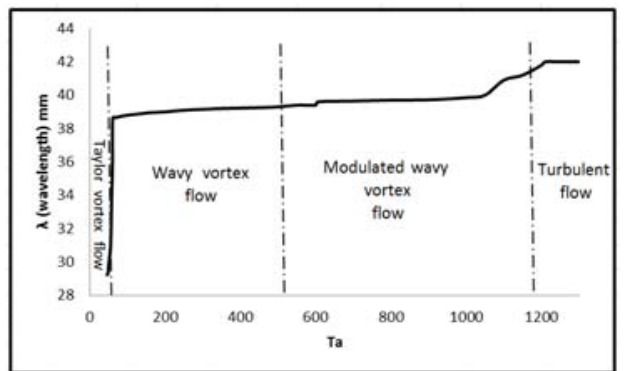

Fig. 27. Wavelength of the Couette-Taylor flow with respect to Taylor numbers

The critical Taylor numbers for transition between different flow regimes and different geometrical conditions can be obtained by present modeling with high accuracy.

The results showed that the wavelength of the flow conFiguration increases sharply at transition from Taylor vortex flow into wavy vortex flow. It is because of the azimuthal wave that is superimposed on Taylor vortices. Also the wavelength of the modulated wavy vortex flow increases very smoothly with respect to wavy vortex flow. Finally, at transtition from modulated wavy vortex flow into turbulent flow, the wavelength of the flow increases with moderate slope. It is because of the turbulent structures. In addition the wavelength of the flow in every flow regime is approximately constant with respect to Taylor numbers.

\section{ACKNOWLEDGMENTS}

This work was supported by the laboratories GEPEA (University of Nantes), LAMIH
(University of Valenciennes), the "Agence Nationale de la Recherche (ANR), France" by the grant $\mathrm{n}^{\circ}$ ANR-08-BLAN-0184-02, and Isfahan University of Technology, Iran. These supports are gratefully acknowledged.

\section{REFERENCES}

Bazilevs, Y., Akkerman, I. (2010) Large Eddy Simulation of turbulent Taylor-Couette flow using isogeometric analysis and the residual-based variational multiscale method, Journal of Computational Physiscs, 229, 3402-3414.

Berrich, E. (2011) Analyse expérimentale des interactions écoulement - paroi par PIV et polarographie en utilisant des sondes multisegmentées : Application à un rhéomètre plan-plan et un système de Couette-Taylor, Ph.D. Thesis, University of Nantes, France.

Berrich, E., Aloui, F. and Legrand, J. (2012) On the stability of Taylor-Couette flow with axial flow, ASME FEDSM, 7th Symposium on flow manipulation and cetive Control, Vol. 1, Parts A and B, FEDSM2012-72235, pp. 899-908, Rio Grande, Puerto Rico, USA, July 8-12.

Cohen, S., Marom, D. M. (1991) Analysis of a rotating annular reactor in the vortex flow regime, chemical Engineering Science, 46, 123-134.

Couette, M. (1890) Études sur le frottement des liquids, Ann. Chim. Phys. 6, Ser. 21, 433-510.

Dong, S. (2008) Turbulent flow between counterrotating concentric cylinders: a direct numerical simulation study. Journal Fluid Mechanics, 615, 371-399.

Holeschovsky, U. B. and Conney, C. L., (1991) Quantitative description of ultrafiltration in a rotating filtration device, A. I. Ch. E. Journal 37, 8, 12-19 (1991).

Mallock, A. (1888) Determination of the viscosity of water, Proc. Royal Soc. London, 45,126-132.

Newton, I. (1934) The Motion of Bodies, Principia: Vol. 1, F. Cajori, ed., U. Calif. Press, Berkeley, 385386.

Poncet, S., Soghe, R., Bianchini, C., Viazzo, S. and Aubert, A. (2013) Turbulent Couette-Taylor flows with endwall effects: A numerical Benchmark, International Journal of Heat and Fluid Flow.

Rayleigh, L. (1916) on the dynamics of revolving fluids, Proc. Royal Soc. A, 93:148-154.

Stokes, G. (1880) Mathematical and Physical Papers, 1, Cambridge Univ. Press, Cambridge, England, 102-104.

Taylor, G. I. (1923) Stability of a viscous liquid contained between two rotating cylinders, Philos. Trans. R. Soc. London, Ser. A, 223:289-343.

Wereley S. T., Lueptow, R. M. (1998) Spatiotemporal character of non-wavy and wavy TaylorCouette flow, Journal of Fluid Mechanics., 364:5980 . 
M. Monfared et al. /JAFM, Vol. 9, Special Issue 1, pp. 49-57, 2016. 